\title{
The prognostic and predictive value of Tregs and tumor immune subtypes in postmenopausal, hormone receptor-positive breast cancer patients treated with adjuvant endocrine therapy: a Dutch TEAM study analysis
}

\author{
C. C. Engels • A. Charehbili $\cdot$ C. J. H. van de Velde $\cdot$ E. Bastiaannet $\cdot$ \\ A. Sajet $\cdot$ H. Putter $\cdot$ E. A. van Vliet $\cdot$ R. L. P. van Vlierberghe $\cdot$ V. T. H. B. M. Smit $\cdot$ \\ J. M. S. Bartlett $\cdot$ C. Seynaeve $\cdot$ G. J. Liefers $\cdot$ P. J. K. Kuppen
}

Received: 7 November 2014/ Accepted: 5 January 2015/Published online: 24 January 2015

(C) The Author(s) 2015. This article is published with open access at Springerlink.com

\begin{abstract}
Evidence exists for an immunomodulatory effect of endocrine therapy in hormone receptor-positive $(\mathrm{HR}+\mathrm{ve})$ breast cancer $(\mathrm{BC})$. Therefore, the aim of this study was to define the prognostic and predictive value of tumor immune markers and the tumor immune profile in $\mathrm{HR}+\mathrm{ve} \mathrm{BC}$, treated with different endocrine treatment regimens. 2,596 Dutch TEAM patients were treated with 5 years of adjuvant hormonal treatment, randomly assigned to different regimens: 5 years of exemestane or sequential treatment (2.5 years of tamoxifen-2.5 years of exemestane). Immunohistochemistry was performed for HLA class I, HLA-E, HLA-G, and FoxP3. Tumor immune subtypes (IS) (low, intermediate \& high immune susceptible) were determined by the effect size of mono-immune
\end{abstract}

C. C. Engels and A. Charehbili contributed equally toward this study.

Electronic supplementary material The online version of this article (doi:10.1007/s10549-015-3269-7) contains supplementary material, which is available to authorized users.

C. C. Engels - A. Charehbili - C. J. H. van de Velde .

E. Bastiaannet - A. Sajet . E. A. van Vliet .

R. L. P. van Vlierberghe · G. J. Liefers · P. J. K. Kuppen ( $₫)$ Department of Surgery, Leiden University Medical Center, Albinusdreef 2, 2300RC Leiden, The Netherlands

e-mail: p.j.k.kuppen@lumc.nl

A. Charehbili

Department of Clinical Oncology, Leiden University Medical Center, Albinusdreef 2, 2300RC Leiden, The Netherlands

\section{E. Bastiaannet}

Department of Gerontology, Leiden University Medical Center, Albinusdreef 2, 2300RC Leiden, The Netherlands

H. Putter

Department of Statistics, Leiden University Medical Center,

Albinusdreef 2, 2300RC Leiden, The Netherlands markers on relapse rate. Patients on sequential treatment with high level of tumor-infiltrating FoxP3 + cells had significant $(p=0.019$, HR $0.729,95 \%$ CI $0.560-0.949)$ better OS. Significant interaction for endocrine treatment and FoxP3+ presence was seen (OS $p<0.001)$. Tumor IS were only of prognostic value for the sequentially endocrine-treated patients (RFP: $p=0.035$, HR intermediate IS 1.420, $95 \%$ CI 0.878-2.297; HR low IS 1.657, $95 \%$ CI 1.131-2.428; BCSS: $p=0.002$, HR intermediate IS 2.486, $95 \%$ CI 1.375-4.495; HR low IS 2.422, $95 \%$ CI 1.439-4.076; and OS: $p=0.005$, HR intermediate IS 1.509, $95 \%$ CI 0.950-2.395; HR low IS 1.848, $95 \%$ CI 1.277-2.675). Tregs and the tumor IS presented in this study harbor prognostic value for sequentially endocrinetreated $\mathrm{HR}+\mathrm{ve}$ postmenopausal BC patients, but not for solely exemestane-treated patients. Therefore, these markers could be used as a clinical risk stratification tool to guide adjuvant treatment in this BC population.

\author{
V. T. H. B. M. Smit \\ Department of Pathology, Leiden University Medical Center, \\ Albinusdreef 2, 2300RC Leiden, The Netherlands \\ J. M. S. Bartlett \\ Ontario Institute for Cancer Research, Toronto, ON, Canada \\ C. Seynaeve \\ Department of Medical Oncology, Erasmus University Medical \\ Center Cancer Institute, Groene Hilledijk 301, \\ 3075AE Rotterdam, The Netherlands
}


Keywords Breast cancer - Adjuvant endocrine therapy . Tumor immune subtypes - Prognostic and predictive value

\section{Introduction}

Breast cancer (BC) is the most commonly diagnosed female cancer in the developed world and also a leading cause of cancer death, responsible for $14 \%$ of cancer-related deaths in women of the West [1]. Nowadays, BC treatment consists of a combination of locoregional treatment (i.e., surgery and radiotherapy) and systemic therapy (i.e., chemotherapy and hormonal therapy), to concur present and less evident metastasis. In the USA, an increased tendency of adjuvant treatment allocation using genomic expression assays such as Oncotype DX (genomic health, redwood city, CA, USA) and Mammaprint (Agendia, Amsterdam, the Netherlands), providing additional information about the risk of relapse and benefit of adjuvant chemotherapy, is seen [2-4]. However, in the Netherlands, decisions regarding the use of adjuvant systemic therapy in primary BC patients are still mainly based on classical prognostic factors, like lymph node status, tumor size, and grade, hormone receptor (HR) and human epidermal growth factor receptor 2 (HER2) expression [5]. However, currently these do not provide optimal risk stratification, resulting in over- and under treatment of certain patients. There is evidence that a host's cellular immune response plays a pivotal role in controlling tumor progression through a number of immunological mechanisms, involving classical human leukocyte antigen (HLA) class I and non-classical HLA-E and HLA-G expression by the tumor, and presence of tumor-infiltrating cytotoxic T cells (CTL), Natural Killer (NK) cells, and regulatory $\mathrm{T}$ cells (Tregs) [6-11], suggesting that complex interactions take place between breast tumor cells and immune cells [12]. Valuable prognostic interactions reported are those between classical HLA class I and Tregs, where loss of HLA class I in combination with the presence of Treg in the tumor microenvironment resulted in a worse patient's outcome, and also the interaction between classical HLA class I, HLA-E, and HLA-G tumor expression, where HLA-E and HLA-G expression resulted in worse patient outcome in the cooccurrence of loss of classical HLA class I on the tumor surface $[8,9,12]$. Together, this emphasizes the importance of research on combinations of markers of immune surveillance together with markers of tumor immune escape.

Our group previously constructed breast tumor immune subtypes (IS) by combining markers of immune surveillance together with markers of tumor immune escape, based on a biological rationale [13]. Data revealed strong associations with patient outcome whereby tumors defined as highly susceptible to immune attack showed favorable clinical outcome compared to patients with tumors harboring a low immune susceptibility profile, independent of known clinicopathological parameters [13]. In the current study, we used another approach to define tumor IS. Tumor immune mono-markers in Dutch postmenopausal hormone-sensitive $\mathrm{BC}$ patients from the Tamoxifen and Exemestane Adjuvant Multicenter (TEAM) trial were correlated to clinical outcome. Subsequently, we designed tumor immune subtypes based on statistical effect sizes of the immune mono-markers on relapse rate.

It has already been shown that tumor-infiltrating lymphocytes (TILs) act as an independent predictor of response to chemotherapy treatment [14-16]. Elaborating on this result, evidence also exists for an immunomodulatory effect of tamoxifen; it is thought that tamoxifen induces a shift from cellular (T-helper 1) to humoral (Thelper 2) immunity [17]. Given the fact that T-helper 1 immunity is essential for anti-tumor immune response, a tamoxifen-induced shift away from cellular immunity may represent a significant step in tumor development. This would hamper the cytotoxic effect of tamoxifen and possibly explain the differential effect of aromatase inhibitors versus tamoxifen on clinical outcome [17-19].

The aim of our current study was therefore to investigate the difference in prognostic value of tumor IS in relation with type of hormonal treatment received in HR+ve, postmenopausal $\mathrm{BC}$ patients.

\section{Patients and methods}

\section{Patients and tumors}

Eligibility criteria for the TEAM study have been previously described [20]. In brief, patients were postmenopausal and had HR+ve early BC diagnosed between 2001 and 2006. Patients with bilateral tumors or prior history of cancer were excluded. Patients were randomly assigned in a 1:1 ratio to either exemestane, $25 \mathrm{mg}$ daily for 5 years, or sequential therapy consisting of tamoxifen $20 \mathrm{mg}$ daily for 2.5 years followed by exemestane $25 \mathrm{mg}$ daily for another 2.5 years [20].

Medical-ethical approval was obtained and the study was conducted in accordance with the Declaration of Helsinki. All TEAM patients gave informed consent prior to enrollment in the study. Surgically resected, formalin-fixed, paraffin-embedded (FFPE) tumor samples of the Dutch TEAM patients $(n=2596)$ were used. All samples were handled in a coded fashion, according to national ethical guidelines ("Code for Proper Secondary Use of Human Tissue", Dutch Federation of Medical Scientific Societies).

Data were centrally collected at the Datacenter of the Department of Surgery of the Leiden University Medical Center. For all patients, the following data were known: age at diagnosis, histological tumor grade, HR status, tumor size and nodal stage, type(s) of local and systemic treatment, date and type of disease recurrence, and death 
and follow-up data. Reporting of the biomarkers was done according to the REMARK criteria [21].

Immunohistochemistry

Immunohistochemical staining was performed on $4 \mu \mathrm{m}$ FFPE Tissue Micro Array sections consisting of breast cancer tissue of the Dutch TEAM patients (three $0.6 \mathrm{~mm}^{2}$ tumor tissue punches per patient) [22]. The tissue sections were stained according to the previously described protocol [9]. Sections were incubated at room temperature over night with mouse monoclonal antibodies HCA2 and HC10 (anti-HLA-A and anti-HLAB/C, respectively) [9, 23] for the detection of classical HLA class I on the tumor cell surface. Non-classical HLA class I staining was performed using mouse monoclonal antibodies against HLA-E (MEM-E/02 Clone (sc-51621, Santa Cruz biotechnology, Dallas, Texas) and HLA-G (4H84 Clone (sc-21799, Santa Cruz Biotechnology, Dallas, Texas) [8]. Mouse monoclonal antibodies against FoxP3 (clone 236A/E7 (ab20034, Abcam, Cambridge, United Kingdom)) were used to identify Tregs [9]. All slides were stained simultaneously to avoid inter-assay variation.

\section{Evaluation of immunostaining}

Microscopic quantification of positive tumor cells for HCA2, HC10, HLA-E, and HLA-G was performed in a blinded manner by two independent observers (C.C.E., A.S. and A.v.V). The scores of the three tissue cores were averaged. For HCA2 and HC10, the percentage of tumor cells with membranous staining was assessed. Classical HLA class I expression status was determined according to the standard set by the International HLA and Immunogenetics Workshop [24]. According to this standard, HCA2 and HC10 staining were scored in two categories: score 1 (0-5\% of tumor cells positively stained) or score 2 (5-100\% of tumor cells positively stained). Three groups were defined for classical HLA class I expression: HLA class I loss (both HCA2 and HC10 scored 0-5 \%); HLA class I down-regulation (either HCA2 or HC10 scored 0-5 \%); and HLA class I expression (both HCA2 and HC10 scored 5-100 \%) [9]. For non-classical HLA class I markers, both HLA-E and HLA-G were scored based on the percentage of tumor cells with membranous staining and recategorized in a binary manner. Any specific staining of tumor cells was considered positive and no staining was considered negative for HLA-G. HLA-E expression was divided into quartiles, of which the first quartile was categorized as low HLA-E expression and subsequent quartiles ( $>$ first quartile) as high. FoxP3 + nuclear presence per $\mathrm{mm}^{2}$ in tumor epithelium and surrounding stroma tissue was identified with the use of a Panoramic Midi scanner (3DHistech, Hungary) by means of an automated positive cell count analysis using AxioVision 4.6 (Carl Zeiss Vision, Jena, Germany). FoxP3 + presence was scored by two categories: low ( $\leq 49$ positive cells) and high ( $>49$ positive cells) Treg infiltration per $\mathrm{mm}^{2}$, based on the median value.

\section{Statistical analysis}

Statistical analyses were performed using statistical package SPSS (version 20.0 for Windows, IBM SPSS statistics). Patients whose tumor material was lost during staining procedure were excluded from analyses. Cohen's kappa coefficient was used to assess inter-observer agreement in quantification of HCA2, HC10, HLA-E, and HLA-G. As BC relapse strongly influences survival rates of $\mathrm{BC}$ patients, we designed tumor IS, by combining classical HLA-I, HLA-E, and HLA-G, and FoxP3, based on the regression coefficient of these mono-markers in the Cox-regression using relapse-free period (RFP) as clinical endpoint for all tumor samples. The regression coefficient was used for IS configuration because different modes of immunohistochemical scoring were used for the different markers, making a simple additive approach for the individual scores to construct the IS undesirable. Furthermore, usage of the regression coefficient results in accounting for an accurate degree of impact of an immunohistochemical score on clinical outcome. The regression coefficient value, indicating either negative or positive clinical effect, served as a penalty or bonus (in case of a negative or positive slope, respectively). All regression coefficients (for HLA-I, HLA-E, HLA-G and FoxP3 +) were added up to construct the final score per patient. Ultimately, three groups: low, intermediate, and high immune-susceptible tumor types were constructed based on tertile $(\leq 33,>33, \leq 67$ and $>67 \%$ ) cut-off points of the final score.

The $\chi^{2}$ test was used to evaluate associations between the tumor immune mono-markers, and also between clinicopathological parameters and tumor immune monomarkers and tumor IS. The clinical endpoints were RFP, defined as time from date of randomization in the TEAMtrial until any recurrence (locoregional recurrence and/or a distant recurrence, whichever came first), breast cancerspecific survival (BCSS), defined as time from date of randomization until death due to $\mathrm{BC}$, and overall survival (OS), defined as time from randomization until death by any reason. The Kaplan-Meier method was used for survival plotting and log-rank test for RFP, BCSS, and OS curve comparison. Cox proportional hazard analysis was used for univariate analysis and was additionally adjusted for clinically relevant confounders (age, pathological tumor and nodal stage, tumor grade, histology, and treatment). All analyses were stratified for hormonal regimen (exemestane or sequential regimen). Interaction between endocrine treatment and tumor IS was tested in a multivariable model. 


\section{Results}

Patient and tumor characteristics

The Dutch TEAM cohort consists of 2596 postmenopausal non-metastasized BC patients with a median age of 65 years (range 38-91 years). Median follow-up of patients was 5.9 years. Clinicopathological and treatment characteristics in relation with tumor IS are shown in Table 1. Only for radiotherapy a significant difference (Chi-square test, $p=0.045$ ) was seen between tumor IS, showing less radiotherapy treatment for intermediate tumor IS compared to low and high tumor IS. Substantial agreement $(K \geq 0.6)$ was observed for quantification of all immunohistochemical stainings.

Classical HLA-I expression and association with prognosis

Microscopic quantification for classical HLA-I was successful in $73 \%(1891 / 2596)$ of tumors $(79 \%(2042 / 2596)$ for HCA2 and $80 \%(2083 / 2596)$ for HC-10). Classical HLA-I loss was found in $16 \%(298 / 1891)$, down-regulation in $27 \%(513 / 1891)$, and expression in $57 \%$ (1080/ 1891)(Supplementary Table 1A). In the analyses stratified for endocrine treatment, no significant difference in outcome was seen for HLA-I expression in RFP, BCSS, or OS (Supplementary Table 2A).

HLA-E and HLA-G expression and association with prognosis

Successful staining for HLA-E was obtained in $74 \%$ of tumors, and in $79 \%$ for HLA-G. Low HLA-E was found in $26 \%(495 / 1914)$ and high expression in $74 \%(1419 / 1914)$ of the patients, whereas absence of HLA-G was found in $76 \%(1558 / 2042)$ and expression in $24 \%(484 / 2042)$ of the patients (Supplementary Table 1B). Neither of the two immune markers showed significant association with clinical outcome when stratified for endocrine treatment received (Supplementary Table 2B and 2C).

Presence of FoxP3 + cells and association with prognosis

Automated positive cell count was successful in $93 \%$ $(2426 / 2596)$ of tumors for FoxP3 + cells. Low ( $\leq$ median value of 49 cells) number of positive cells was seen in $51 \%(1241 / 2426)$ and high number ( $>$ median of 49 positive cells) in $49 \%(1185 / 2426)$ of the patients (Supplementary Table 1A). Patients on sequential hormonal therapy showed a significant (univariate: $p=0.026$, multivariate: $p=0.019$, HR: $0.729,95 \%$ CI $0.560-0.949$ ) preferential outcome for high FoxP3+ presence in OS, but not for RFP or BCSS. No association with clinical outcome was seen for patients in the exemestane-only treated arm (univariate OS: $p=0.138, \quad \mathrm{HR} \quad 0.821,95 \% \quad \mathrm{CI}$ : 0.633-1.065) (Supplementary Table 2D). The multivariable interaction model showed a significant predictive effect for endocrine treatment and FoxP3+ presence ( $p$ value $\mathrm{OS}<0.001)$ in OS.

Tumor immune subtypes and association with prognosis

In view of recent evidence stating that the interaction between tumor cells and cells of the immune system is multifaceted and complex [13], we hypothesized that combined analyses of immune markers may better reflect a patients' outcome by taking into account the interaction between tumor cells and cells of the immune system. First, when the four mono-markers were tested in relation to one another in the Chi-square test, results showed a significant association between all four mono-markers (Chi-square test, $p$ values: all $<0.001$, data not shown). No difference in distribution was observed for the defined risk groups in the two hormonal treatment arms $(p=0.726)$. Based on the tumor IS model described in the Materials and methods section, which is based on the regression coefficient of the mono-markers in the RFP, high tumor immune susceptibility was characterized by either classical HLA-I expression with HLA-EG presence or absence (HLA-EG absence: both or either HLA-E or HLA-G not expressed; HLA-EG positive: both HLA-E and HLA-G positive) on the tumor surface, known for its activation of Natural Killer (NK) cells [8], or classical HLA-I loss or down-regulation combined with mostly HLA-EG absence. Treg presence was equally distributed in the high IS tumor subtypes. Great variability in Treg presence was also seen in the low and intermediate tumor IS (Supplementary Table 3). The tumor IS showed significant preference for the high immune-susceptible tumor types for clinical outcome (RFP: $p=0.002$, HR intermediate (vs. high) tumor IS 1.539, $95 \%$ CI 1.088-2.178; HR low (vs. high) tumor IS 1.634, $95 \%$ CI 1.235-2.163; BCSS: $p<0.001$, HR intermediate (vs. high) tumor IS: $2.119,95 \%$ CI 1.368-3.283; HR low (vs. high) tumor IS 2.103, $95 \%$ CI 1.456-3.038); OS: $p=0.002$, HR intermediate (vs. high) tumor IS 1.471, $95 \%$ CI 1.065-2.032; HR low (vs. high) tumor IS 1.602, $95 \%$ CI 1.235-2.077) (Fig. 1).

Immune subtypes and adjuvant endocrine treatment

Significant differences were seen for RFP, BCSS, and OS in the sequentially endocrine-treated patient group when stratified for adjuvant hormonal treatment. Again, all 
Table 1 Patient and tumor characteristics

\begin{tabular}{|c|c|c|c|c|c|c|c|}
\hline & \multicolumn{2}{|c|}{ High immune subtype } & \multicolumn{2}{|c|}{ Intermediate immune subtype } & \multicolumn{2}{|c|}{ Low immune subtype } & \multirow[t]{2}{*}{$p$ value } \\
\hline & $N=501$ & $\%$ & $N=318$ & $\%$ & $N=817$ & $\%$ & \\
\hline \multicolumn{8}{|l|}{ Age } \\
\hline$<65$ & 259 & 51.7 & 164 & 51.6 & 425 & 52.0 & \multirow[t]{3}{*}{0.988} \\
\hline$\geq 65$ & 242 & 48.3 & 154 & 48.4 & 392 & 48.0 & \\
\hline Missing & 0 & & 0 & & 0 & & \\
\hline \multicolumn{8}{|l|}{ pT stage } \\
\hline $\mathrm{T} 1$ & 227 & 45.4 & 126 & 39.6 & 385 & 47.2 & \multirow[t]{4}{*}{0.218} \\
\hline $\mathrm{T} 2$ & 244 & 48.8 & 169 & 53.1 & 387 & 47.4 & \\
\hline T3-4 & 29 & 5.8 & 23 & 7.2 & 44 & 5.4 & \\
\hline Missing & 1 & & 0 & & 1 & & \\
\hline \multicolumn{8}{|l|}{ pN stage } \\
\hline No & 143 & 28.5 & 107 & 33.6 & 277 & 33.9 & \multirow[t]{4}{*}{0.373} \\
\hline N1 & 319 & 63.7 & 192 & 60.4 & 490 & 60.0 & \\
\hline $\mathrm{N} 2-3$ & 39 & 7.8 & 19 & 6.0 & 49 & 6.1 & \\
\hline Missing & 0 & & 0 & & 1 & & \\
\hline \multicolumn{8}{|l|}{ Grade } \\
\hline I & 66 & 13.9 & 55 & 18.5 & 98 & 12.6 & \multirow[t]{4}{*}{0.100} \\
\hline II & 222 & 46.8 & 134 & 45.1 & 348 & 44.8 & \\
\hline III & 186 & 39.3 & 108 & 36.4 & 330 & 42.6 & \\
\hline Missing & 27 & & 21 & & 41 & & \\
\hline \multicolumn{8}{|l|}{ Histology } \\
\hline Ductal & 391 & 78.5 & 249 & 78.6 & 664 & 81.8 & \multirow[t]{5}{*}{0.495} \\
\hline Lobular & 65 & 13.1 & 40 & 12.6 & 79 & 9.7 & \\
\hline Mixed & 18 & 3.6 & 14 & 4.4 & 37 & 4.6 & \\
\hline Other & 24 & 4.8 & 14 & 4.4 & 32 & 3.9 & \\
\hline Missing & 3 & & 1 & & 5 & & \\
\hline \multicolumn{8}{|l|}{ Operation } \\
\hline Mastectomy & 263 & 52.5 & 183 & 57.5 & 434 & 53.1 & \multirow[t]{3}{*}{0.318} \\
\hline BCS & 238 & 47.5 & 135 & 42.5 & 383 & 46.9 & \\
\hline Missing & 0 & & 0 & & 0 & & \\
\hline \multicolumn{8}{|l|}{ Radiotherapy } \\
\hline Yes & 318 & 63.5 & 174 & 54.9 & 500 & 61.2 & \multirow[t]{3}{*}{0.045} \\
\hline No & 183 & & 143 & & 317 & & \\
\hline Missing & 0 & & 1 & & 0 & & \\
\hline \multicolumn{8}{|l|}{ Chemotherapy } \\
\hline Yes & 129 & 25.7 & 102 & 32.2 & 247 & 30.2 & \multirow[t]{3}{*}{0.097} \\
\hline No & 372 & 74.3 & 215 & 67.8 & 570 & 69.8 & \\
\hline Missing & 0 & & 1 & & 0 & & \\
\hline \multicolumn{8}{|l|}{ Endocrine therapy } \\
\hline EXE & 257 & 51.3 & 154 & 48.4 & 410 & 50.2 & \multirow[t]{3}{*}{0.726} \\
\hline $\mathrm{TAM} \rightarrow \mathrm{EXE}$ & 244 & 48.7 & 164 & 51.6 & 407 & 49.8 & \\
\hline & 0 & & 0 & & 0 & & \\
\hline
\end{tabular}

EXE exemestane, TAM tamoxifen

outcomes are in favor of high tumor immune susceptibility (RFP: sequential treatment: $p=0.035$, HR intermediate IS (versus high) $1.420,95 \%$ CI $0.878-2.297$; HR low IS (versus high): 1.657, $95 \%$ CI 1.131-2.428; BCSS:sequential treatment: $p=0.002$, HR intermediate IS (vs. high) 2.486, $95 \%$ CI 1.375-4.495; HR low IS (vs. 

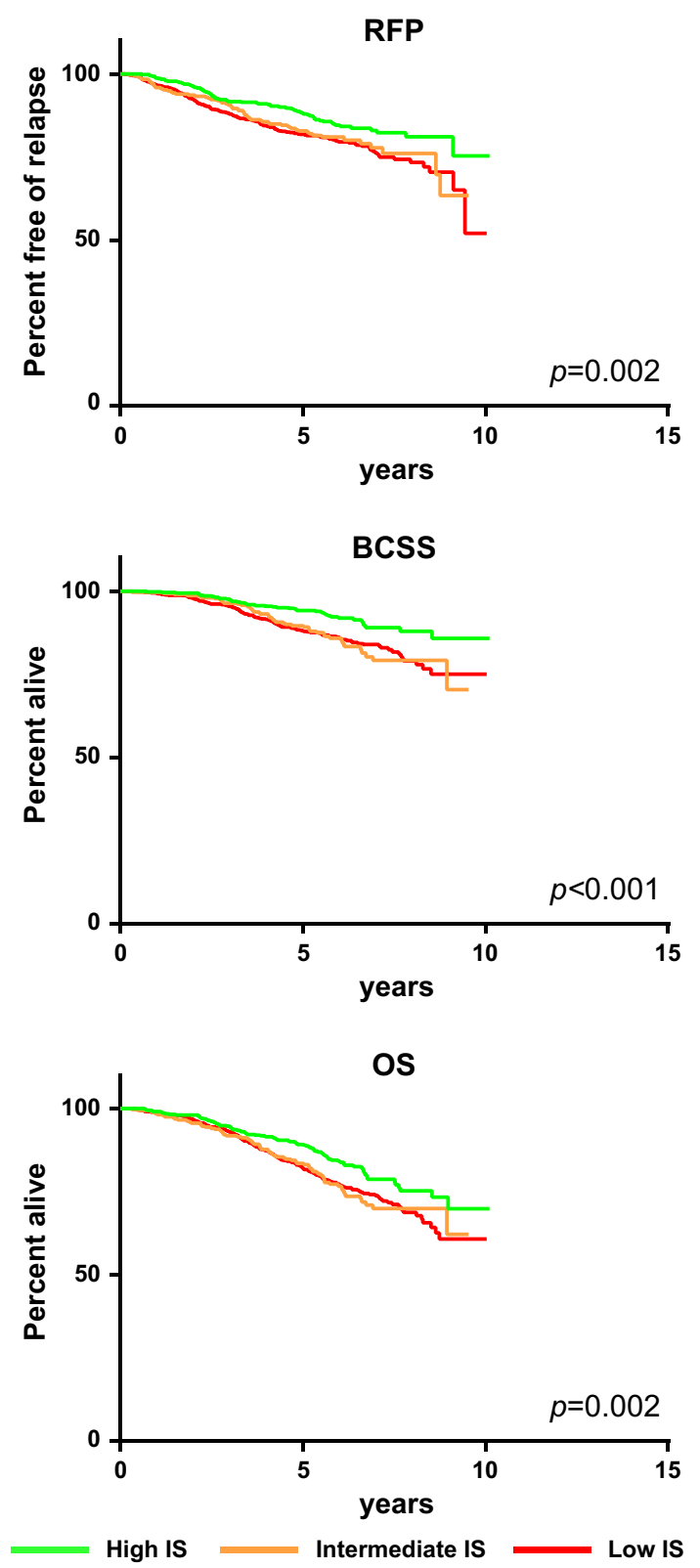

Fig. 1 Tumor immune subtypes [high, intermediate, and low tumor immune subtypes (IS)] in relation with clinical outcome parameters: relapse-free period (RFP), breast cancer-specific survival (BCSS), and overall survival (OS), shown with corresponding adjusted (age, pT stage, pN stage, tumor grade, histology, surgery type, chemotherapy, radiotherapy, and endocrine therapy) $p$ values

high) 2.422, $95 \%$ CI 1.439-4.076; and OS:sequential treatment: $p=0.005$, HR intermediate IS (vs. high) 1.509, $95 \%$ CI 0.950-2.395; HR low IS (versus high) 1.848, $95 \%$ CI 1.277-2.675) (Table 2; Fig. 2). No prognostic value was seen for the solely exemestane-treated patients. A statistical trend was seen for the interaction between endocrine treatment and tumor IS in the multivariable interaction model ( $p$ value RFP: 0.15, BCSS: 0. 19 and OS: $0.17)$.

\section{Discussion}

Evidence is building for an increasingly important role of tumor-immune interaction with regard to clinical outcome of cancer patients [25]. To our knowledge, this is the first study reporting on the effect of endocrine treatment on the prognostic value of Treg cells and tumor IS in a HR+ve $\mathrm{BC}$ cohort.

Our data suggest a positive effect of Treg presence on overall survival outcome in the sequentially endocrinetreated patient group, which is further supported by a highly significant interaction term for endocrine treatment and Treg presence. This could possibly be explained by recent data indicating that Tregs harbor a dual role in cancer, suppressing anti-tumor immune response (inducible Treg) and suppressing inflammation which is known to promote carcinogenesis (natural Treg) [26, 27]. These same studies suggest that the clinical and prognostic significance of Tregs in cancer depends on its environmental factors. Our investigated patient population harbors a number of pro-inflammatory risk factors, namely a postmenopausal status which is known to be associated with systemic inflammation, and HR+ve breast tumors [28]. Assuming that HR+ve tumors attract higher estrogen levels in and around the tumor due to an increased tendency of estrogen binding, we hypothesize that this estrogen-rich environment leads to higher Adenosine Deaminase Gene expression, which in turn is responsible for the degradation of Adenosine (ADO), a potent anti-inflammatory agent [29, 30]. This presumed high inflammatory state in our patient population would assume a preference for natural Tregs, explaining the positive effect of high FoxP3+ presence in the tumors and the loss of prognostic significance in solely exemestane-treated patients, as aromatase inhibition leads to lower estrogen levels, which will diminish ADO degradation.

For BC patients treated with sequential endocrine therapy, the tumor IS bare a strong independent significant prognostic value for BC-specific survival and also, although to a lesser degree, for relapse rate and overall survival, while this association was not seen for patients treated solely with aromatase inhibition for five consecutive years. These data might imply that the immune profile of the breast tumor in sequentially endocrine-treated breast cancer patients could predict BC death and overall death in $\mathrm{HR}+\mathrm{ve}$ breast disease, and thus additional adjuvant therapy, such as chemotherapy and radiotherapy, could be optimally allocated based on this prognostic indicator. Since no prognostic effect was noted for the tumor IS in the solely exemestane-treated patient population, the question remains whether there would be any benefit of additional adjuvant treatment for these patients, suggesting that currently we might have obtained the best attainable clinical 
Table 2 Cox univariate and multivariate analysis for overall survival (OS) and relapse-free survival (RFS) stratified for different endocrine therapy regimens for tumor immune subtypes classified into 3 groups

\begin{tabular}{|c|c|c|c|c|c|c|c|c|c|c|}
\hline \multirow[t]{2}{*}{ Outcome } & \multirow[t]{2}{*}{ Hormone therapy } & \multirow[t]{2}{*}{ Immune subtype } & \multirow[t]{2}{*}{$\mathrm{N}$} & \multicolumn{3}{|c|}{ Univariate } & \multicolumn{4}{|c|}{ Multivariate $^{\mathrm{a}}$} \\
\hline & & & & HR & $95 \% \mathrm{CI}$ & $p$ & HR & $95 \% \mathrm{CI}$ & $p$ & Interaction $p$ \\
\hline \multirow[t]{3}{*}{ RFP } & \multirow[t]{3}{*}{ EXE } & High & 257 & 1.00 & & 0.113 & - & - & - & 0.15 \\
\hline & & Intermediate & 154 & 1.556 & $0.958-2.526$ & & & & & \\
\hline & & Low & 410 & 1.464 & $0.988-2.171$ & & & & & \\
\hline \multirow[t]{3}{*}{ RFP } & \multirow[t]{3}{*}{$\mathrm{TAM} \rightarrow \mathrm{EXE}$} & High & 244 & 1.00 & & 0.086 & 1.00 & & 0.035 & \\
\hline & & Intermediate & 164 & 1.343 & $0.850-2.122$ & & 1.420 & $0.878-2.297$ & & \\
\hline & & Low & 407 & 1.520 & $1.049-2.203$ & & 1.657 & $1.131-2.428$ & & \\
\hline \multirow[t]{3}{*}{ BCSS } & \multirow[t]{3}{*}{ EXE } & High & 257 & 1.00 & & 0.261 & - & - & - & 0.19 \\
\hline & & Intermediate & 154 & 1.482 & $0.812--2.708$ & & & & & \\
\hline & & Low & 410 & 1.465 & $0.907-2.367$ & & & & & \\
\hline \multirow[t]{3}{*}{ BCSS } & \multirow[t]{3}{*}{ TAM $\rightarrow$ EXE } & High & 244 & 1.00 & & 0.002 & 1.00 & & 0.0 & \\
\hline & & Intermediate & 164 & 2.486 & $1.375-4.495$ & & 2.848 & $1.509-5.375$ & 01 & \\
\hline & & Low & 407 & 2.422 & $1.439-4.076$ & & 2.869 & $1.651-4.984$ & & \\
\hline \multirow[t]{3}{*}{ OS } & \multirow[t]{3}{*}{ EXE } & High & 257 & 1.00 & & 0.204 & - & - & - & 0.17 \\
\hline & & Intermediate & 154 & 1.428 & $0.925-2.205$ & & & & & \\
\hline & & Low & 410 & 1.311 & $0.924-1.858$ & & & & & \\
\hline \multirow[t]{3}{*}{ OS } & \multirow[t]{3}{*}{$\mathrm{TAM} \rightarrow \mathrm{EXE}$} & High & 244 & 1.00 & & 0.024 & 1.00 & & 0.005 & \\
\hline & & Intermediate & 164 & 1.531 & $0.993-2.362$ & & 1.509 & $0.950-2.395$ & & \\
\hline & & Low & 407 & 1.636 & $1.144-2.341$ & & 1.848 & $1.277-2.675$ & & \\
\hline
\end{tabular}

TAM tamoxifen, EXE exemestane

${ }^{a}$ Adjusted for age, pT stage, pN stage, tumor grade, histology, surgery type, chemotherapy, and radiotherapy

outcome with five consecutive years of exemestane treatment, even for the low tumor immune-susceptible HR+ve patient population. However, the multivariable interaction term for endocrine treatment and breast tumor immune subtypes hinted to a possible statistical trend for clinical outcome. The lack of significance in this test could be explained by the limited power of the statistical interaction test and also due to the low number of clinical events in our cohort.

In this study, it was hypothesized that high immunesusceptible tumor types, due to a tamoxifen-induced shift from Th1 to Th2 immunity, would have the highest likelihood of showing regression of clinical outcome to mean relapse and survival rates of the overall cohort. Based on the data presented in this manuscript, the difference in prognostic value of tumor immune subtyping between the two endocrine treatment arms cannot be explained by the previously described tamoxifen-driven shift from Th1 to Th2 immunity [17]. In that case, it would be expected that the difference in prognosis between the high immunesusceptible tumor subtype, which is expected to be strongly dependent on cellular Th1 immunity, and the low and intermediate subtypes would be minimized. Reason for this could be that highly immunogenic tumors have the ability to circumvent the inferior immune response caused by the tamoxifen-induced Th1-to-Th2 shift, by means of other immune interactions not requiring Th1 activation. A possible explanation for the loss of prognostic value of the tumor IS in the exemestane-treated patient arm of this cohort could also be Treg dependent. Findings supporting exemestane-induced loss of Treg are published by Chan et al., showing a significant increase in the CD8+/Treg ratio in $\mathrm{ER}+\mathrm{ve}$ patients, responding well to aromataseinhibiting therapy, herewith reflecting the dynamic process in which the host's immune response to tumor antigens changed in consequence of estrogen depletion caused by the aromatase inhibitor [31]. Similarly, Generali et al. observed that FoxP3+ cell counts decreased significantly after letrozole treatment [32]. Therefore, one could hypothesize that in this specific $\mathrm{HR}+\mathrm{ve}$, postmenopausal BC cohort, exemestane-induced loss of highly prognostic Treg cells could lead to equalization of the clinical outcomes of the three tumor IS in the solely exemestanetreated adjuvant treatment arm. If this would be true, one could speculate on the great importance of Treg for inhibition of tumor development in a postmenopausal, HR+ve tumor environment, thereby proposing that under these conditions, HLA-I, HLA-E, and HLA-G seem to merely have a supportive role in relation to Treg cells.

This is the first study that assessed the relation between adjuvant endocrine therapy and the prognostic value of tumor immune markers and tumor IS of postmenopausal 
Fig. 2 Tumor immune subtypes [high, intermediate, and low tumor immune subtypes (IS)] in relation with clinical outcome parameters: relapse-free period (RFP), breast cancer-specific survival (BCSS), and overall survival (OS), shown with corresponding $p$ values (as seen in Table 3)

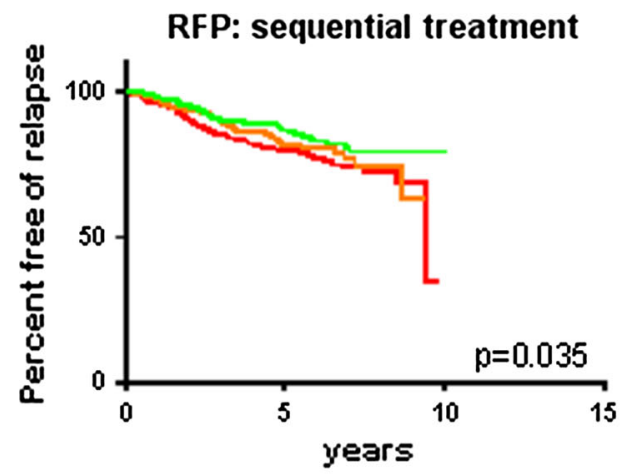

BCSS: sequential treatment

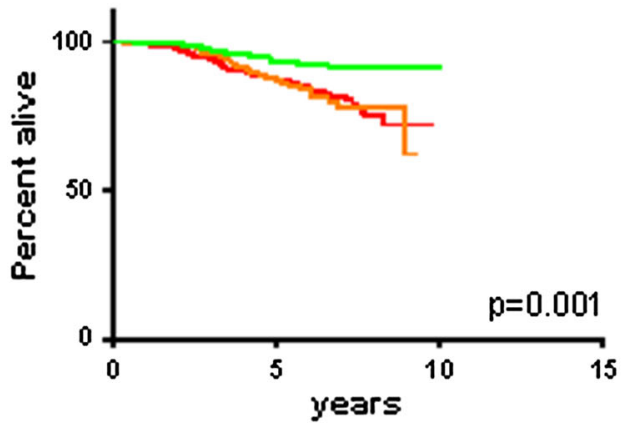

OS: sequential treatment
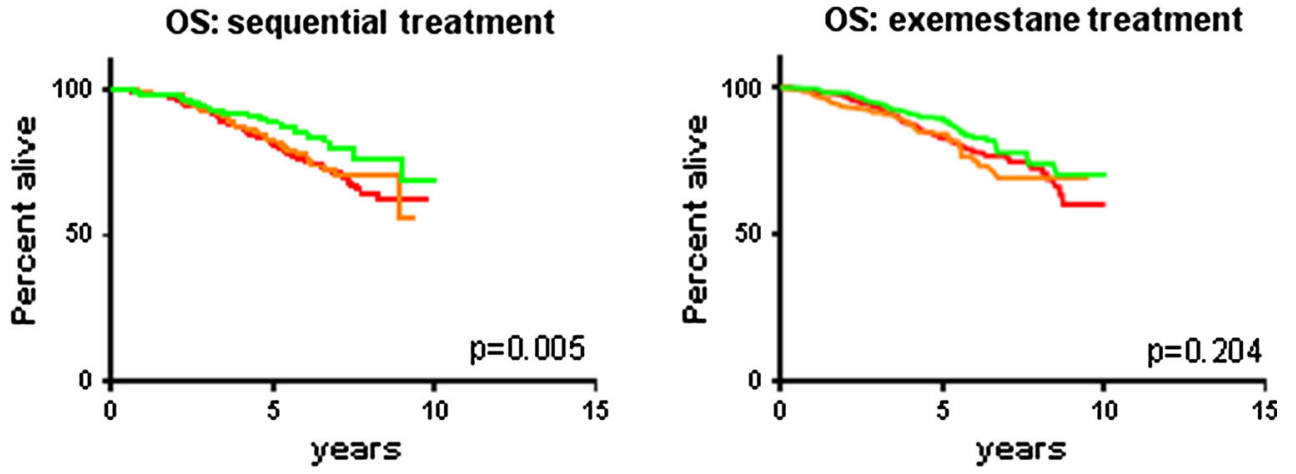

RFP: exemestane treatment

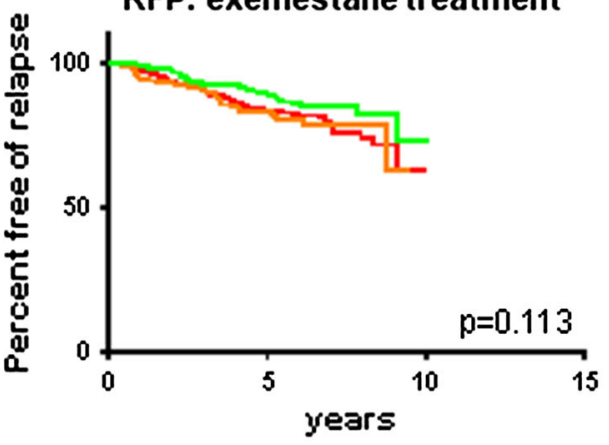

BCSS: exemestane treatment

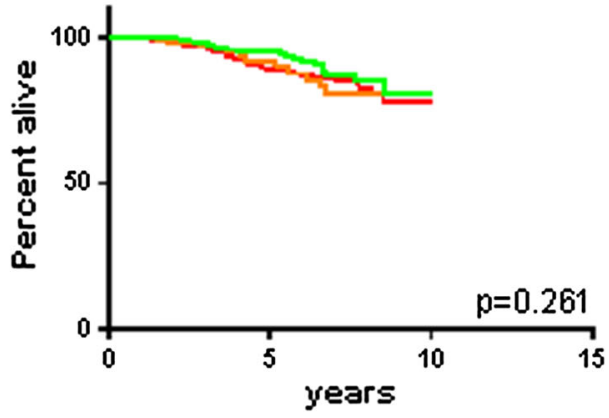

OS: exemestane treatment

- High IS - Intermediate IS - Low IS
$\mathrm{HR}+\mathrm{ve}$, early BC patients. Of course, the external validity of our results should be investigated in other large studies with tumor material available of $\mathrm{HR}+\mathrm{ve} \mathrm{BC}$ patients treated with different hormonal regimens, such as, for example the ATAC, BIG, or IES study [18, 33, 34]. The major strength of this study is the use of data from the TEAM-trial, as this provides well-registered data in a large number of patients. This study, however, also has its limitations. First, one could stress the shortcomings of FoxP3 staining, without co-staining of CD25 and CD4, for the detection of Tregs. Herewith, the margin of error for mistakenly scoring FoxP3+ breast tumor cells is increased [35]. However, based on careful review of the histology of the breast cancer tissue and given the fact that the majority of FoxP3 + cells were seen in the stromal region of the tumor tissue, we can state with reasonable certainty that the majority of positive cells were true Treg cells. Second, there were no standard tumor IS categories available from previous literature. Therefore, we categorized patients by tumor IS based on the regression coefficient of the monomarkers in the Cox-regression using RFP. One could criticize that this is an over-fitted model for RFP, but our results also showed significant association with the other clinical outcome parameters BCSS and OS. Furthermore, our results did not show a difference in the distribution of the tumor IS for the two hormonal treatment arms; nevertheless, results showed a clear significant difference in the prognostic value of the IS based on the hormonal treatment received. Third, patients on sequential hormonal therapy received exemestane after the first 2.5 years of tamoxifen 
treatment. It would be desirable to compare two endocrine treatment regimens, consisting of solely exemestane and solely tamoxifen given for five consecutive years, eliminating the potential immune-modulating effects of endocrine drugs with a different mode of action. Lastly, the immune contributions on clinical outcome described in this manuscript are all based on surgically derived tumor material, assuming that metastasizing cells harbor the same immunogenic characteristics. It should not be ignored that this approach disregards the possible interplay of systemic immune cells which undoubtedly also play a major role in anti-tumor immunity.

In conclusion, when taking into account the difference in associations of the tumor immune markers and tumor IS per endocrine treatment arm, these data partially support the hypothesis of previous manuscripts stating that endocrine treatment harbors an immune-modulating effect [17, 31]. Nonetheless, this study merely showed a statistical trend for interaction between tumor IS and type of endocrine treatment, and a strong interaction for FoxP3 + cells present in the tumor and endocrine treatment, implying that based on the data presented in this manuscript, the difference in prognostic value of tumor immune subtyping between the two endocrine treatment arms cannot be explained by the previously described tamoxifen-driven shift from Th1 to Th2 immunity [17]. In that case, it would be expected that the difference in prognosis between the high immune-susceptible tumor subtype, which is expected to be strongly dependent on cellular Th1 immunity, and the low and intermediate subtypes would be minimized. Reason for this could be that highly immunogenic tumors have the ability to circumvent the inferior immune response caused by the tamoxifen-induced Th1-toTh2 shift, by means of other immune interactions not requiring Th1 activation. A possible explanation for the loss of prognostic value of the tumor IS in the exemestane-treated patient arm of this cohort could also be Treg dependent. Findings supporting exemestane-induced loss of Treg are published by Chan et al., showing a significant increase in the CD8 +/Treg ratio in ER + ve patients, responding well to aromatase-inhibiting therapy, herewith reflecting the dynamic process in which the host's immune response to tumor antigens changed in consequence of estrogen depletion caused by the aromatase inhibitor [31]. Similarly, Generali et al. observed that FoxP3 + cell counts decreased significantly after letrozole treatment [32]. Therefore, one could hypothesize that in this specific HR+ve, postmenopausal BC cohort, exemestane-induced loss of highly prognostic Treg cells could lead to equalization of the clinical outcomes of the three tumor IS in the solely exemestane-treated adjuvant treatment arm. If this would be true, one could speculate on the great importance of Treg for inhibition of tumor development in a postmenopausal, HR + ve tumor environment, thereby proposing that, despite the call for strong immune cell interplay recognition in tumor development, under these specific conditions, HLA-I, HLA-E, and HLA-G seem to merely have a supportive role in relation to Treg cells.

To the best of our knowledge, this is the first study showing different associations in the prognostic value of tumor-infiltrating Tregs and tumor IS with adjuvant endocrine treatment, and thus could be used as a clinical risk stratification tool in sequentially endocrine-treated HR+ve, postmenopausal BC patients. Therewithal, the results of this study add to previous studies on tumor-immune interactions in $\mathrm{BC}[6,13,17,36,37]$. More research is needed to further elucidate this clinically relevant matter.

Acknowledgments The authors kindly thank Prof. Dr. J. Neefjes from the Netherlands Cancer Institute (Amsterdam) for providing the anti-HLA-A and anti-HLAB/C antibodies.

Disclosures The authors have declared no conflicts of interest.

Open Access This article is distributed under the terms of the Creative Commons Attribution Noncommercial License which permits any noncommercial use, distribution, and reproduction in any medium, provided the original author(s) and the source are credited.

\section{References}

1. Parkin DM, Bray F, Ferlay J, Pisani P (2005) Global cancer statistics, 2002. CA Cancer J Clin 55:74-108

2. Paik S, Tang G, Shak S et al (2006) Gene expression and benefit of chemotherapy in women with node-negative, estrogen receptor-positive breast cancer. J Clin Oncol 24:3726-3734

3. van't Veer LJ, Dai H, van de Vijver MJ et al (2002) Gene expression profiling predicts clinical outcome of breast cancer. Nature 415:530-536

4. van de Vijver MJ, He YD, van't Veer LJ et al (2002) A geneexpression signature as a predictor of survival in breast cancer. N Engl J Med 347:1999-2009

5. Goldhirsch A, Wood WC, Gelber RD, Coates AS, Thurlimann B, Senn HJ (2007) Progress and promise: highlights of the international expert consensus on the primary therapy of early breast cancer 2007. Ann Oncol 18:1133-1144

6. Algarra I, Garcia-Lora A, Cabrera T, Ruiz-Cabello F, Garrido F (2004) The selection of tumor variants with altered expression of classical and nonclassical MHC class I molecules: implications for tumor immune escape. Cancer Immunol Immunother 53:904-910

7. Bates GJ, Fox SB, Han C et al (2006) Quantification of regulatory $\mathrm{T}$ cells enables the identification of high-risk breast cancer patients and those at risk of late relapse. J Clin Oncol 24:5373-5380

8. de Kruijf EM, Sajet A, van Nes JG et al (2010) HLA-E and HLA$\mathrm{G}$ expression in classical HLA class I-negative tumors is of prognostic value for clinical outcome of early breast cancer patients. J Immunol 185:7452-7459

9. de Kruijf EM, van Nes JG, Sajet A et al (2010) The predictive value of HLA class I tumor cell expression and presence of intratumoral Tregs for chemotherapy in patients with early breast cancer. Clin Cancer Res 16:1272-1280

10. Liu F, Lang R, Zhao J et al (2011) CD8(+) cytotoxic T cell and FOXP3(+) regulatory $\mathrm{T}$ cell infiltration in relation to breast cancer survival and molecular subtypes. Breast Cancer Res Treat 130:645-655 
11. Mahmoud SM, Paish EC, Powe DG et al (2011) Tumor-infiltrating CD8 + lymphocytes predict clinical outcome in breast cancer. J Clin Oncol 29:1949-1955

12. Galon J, Costes A, Sanchez-Cabo F et al (2006) Type, density, and location of immune cells within human colorectal tumors predict clinical outcome. Science 313:1960-1964

13. de Kruijf EM, Engels CC, van de Water W et al (2013) Tumor immune subtypes distinguish tumor subclasses with clinical implications in breast cancer patients. Breast Cancer Res Treat 142(2):355-364

14. Loi S, Sirtaine N, Piette F et al (2013) Prognostic and predictive value of tumor-infiltrating lymphocytes in a phase III randomized adjuvant breast cancer trial in node-positive breast cancer comparing the addition of docetaxel to doxorubicin with doxorubicinbased chemotherapy: BIG 02-98. J Clin Oncol 31:860-867

15. Gooden MJ, de Bock GH, Leffers N, Daemen T, Nijman HW (2011) The prognostic influence of tumour-infiltrating lymphocytes in cancer: a systematic review with meta-analysis. Br J Cancer 105:93-103

16. Denkert C, Loibl S, Noske A et al (2010) Tumor-associated lymphocytes as an independent predictor of response to neoadjuvant chemotherapy in breast cancer. J Clin Oncol 28:105-113

17. Behjati S, Frank MH (2009) The effects of tamoxifen on immunity. Curr Med Chem 16:3076-3080

18. Cuzick J, Sestak I, Baum M et al (2010) Effect of anastrozole and tamoxifen as adjuvant treatment for early-stage breast cancer: 10-year analysis of the ATAC trial. Lancet Oncol 11:1135-1141

19. Thurlimann B, Keshaviah A, Coates AS et al (2005) A comparison of letrozole and tamoxifen in postmenopausal women with early breast cancer. N Engl J Med 353:2747-2757

20. van de Velde CJ, Rea D, Seynaeve C et al (2011) Adjuvant tamoxifen and exemestane in early breast cancer (TEAM): a randomised phase 3 trial. Lancet 377:321-331

21. McShane LM, Altman DG, Sauerbrei W, Taube SE, Gion M, Clark GM (2006) REporting recommendations for tumor MARKer prognostic studies (REMARK). Breast Cancer Res Treat 100:229-235

22. Bartlett JM, Brookes CL, Robson T et al (2011) Estrogen receptor and progesterone receptor as predictive biomarkers of response to endocrine therapy: a prospectively powered pathology study in the Tamoxifen and Exemestane Adjuvant Multinational trial. J Clin Oncol 29:1531-1538

23. Powell AG, Horgan PG, Edwards J (2012) The bodies fight against cancer: is human leucocyte antigen (HLA) class 1 the key? J Cancer Res Clin Oncol 138:723-728
24. Chew SF, Kanaan C, Tait BD (2007) HLA expression and cancer-14th IHIWS immunohistochemistry quality control exercise exchange results. Tissue Antigens 69(Suppl 1):248-251

25. Khong HT, Restifo NP (2002) Natural selection of tumor variants in the generation of "tumor escape" phenotypes. Nat Immunol 3:999-1005

26. Whiteside TL (2012) What are regulatory $\mathrm{T}$ cells (Treg) regulating in cancer and why? Semin Cancer Biol 22:327-334

27. Whiteside TL (2014) Regulatory T cell subsets in human cancer: are they regulating for or against tumor progression? Cancer Immunol Immunother 63:67-72

28. Baumgarten SC, Frasor J (2012) Minireview: inflammation: an instigator of more aggressive estrogen receptor (ER) positive breast cancers. Mol Endocrinol 26:360-371

29. Cronstein BN (1985) Adenosine, an endogenous anti-inflammatory agent. J Appl Physiol 1994(76):5-13

30. Xie W, Duan R, Safe S (1999) Estrogen induces adenosine deaminase gene expression in MCF-7 human breast cancer cells: role of estrogen receptor-Sp1 interactions. Endocrinology 140:219-227

31. Chan MS, Wang L, Felizola SJ et al (2012) Changes of tumor infiltrating lymphocyte subtypes before and after neoadjuvant endocrine therapy in estrogen receptor-positive breast cancer patients-an immunohistochemical study of $\mathrm{Cd} 8+$ and Foxp3 + using double immunostaining with correlation to the pathobiological response of the patients. Int J Biol Markers 27:e295-e304

32. Generali D, Bates G, Berruti A et al (2009) Immunomodulation of FOXP3 + regulatory $\mathrm{T}$ cells by the aromatase inhibitor letrozole in breast cancer patients. Clin Cancer Res 15:1046-1051

33. Regan MM, Neven P, Giobbie-Hurder A et al (2011) Assessment of letrozole and tamoxifen alone and in sequence for postmenopausal women with steroid hormone receptor-positive breast cancer: the BIG 1-98 randomised clinical trial at 8.1 years median follow-up. Lancet Oncol 12:1101-1108

34. Coombes RC, Hall E, Gibson LJ et al (2004) A randomized trial of exemestane after two to three years of tamoxifen therapy in postmenopausal women with primary breast cancer. N Engl J Med 350:1081-1092

35. Takenaka M, Seki N, Toh U et al (2013) FOXP3 expression in tumor cells and tumor-infiltrating lymphocytes is associated with breast cancer prognosis. Mol Clin Oncol 1:625-632

36. Marin R, Ruiz-Cabello F, Pedrinaci S et al (2003) Analysis of HLAE expression in human tumors. Immunogenetics 54:767-775

37. Rouas-Freiss N, Moreau P, Ferrone S, Carosella ED (2005) HLA$G$ proteins in cancer: do they provide tumor cells with an escape mechanism? Cancer Res 65:10139-10144 